Proceedings of Rigidity Theory and Applications

Traverse City, MI, June 14-18 1998

Fundamental Material Science Series, Plenum

\title{
COMPARISON OF CONNECTIVITY AND RIGIDITY PERCOLATION
}

\author{
Cristian F. Moukarzel and Phillip M. Duxbury \\ Department of Physics/Astronomy and \\ Center for Fundamental Materials Research, \\ Michigan State University, \\ East Lansing, MI 48824-1116
}

\section{INTRODUCTION}

Connectivity percolation has devotees in mathematics, physics and in myriad applications [1-4]. Rigidity percolation is a more general problem, with connectivity percolation as an important limiting case. There is a growing realization that the general rigidity percolation problem exhibits a broader range of fundamental phenomena and has the potential for many new applications of percolation ideas and models. In connectivity percolation, the propagation of a scalar quantity is monitored, while in rigidity the propagation of a vector is, in general, considered. In both cases, one or more conservation laws hold. Moreover, connectivity percolation is rather special and appears to be, in many cases, quite different than other problems in the general rigidity class. We illustrate these differences by comparing connectivity and rigidity percolation in two cases which are very well understood, namely diluted triangular lattices $[5,6]$ and trees [7].

A large part of the intense fundamental interest in percolation is due to the fact that percolation is like a phase transition, in the sense that there is a critical point (critical concentration) and non-trivial scaling behavior near the critical point [1-4]. This analogy carries over to the rigidity percolation problem. However, although the connectivity percolation problem is usually second order (including on trees), the rigidity percolation problem is first order in mean field theory and on trees [7]. However on triangular lattices, both connectivity and rigidity percolation are second order, though they are in different universality classes $[5,6]$. The emphasis of most of the analysis in the literature and in this presentation is the behavior at and near the critical point.

In this paper, we discuss (Section 2) ideas which apply to connectivity and rigidity percolation on diluted lattices. In Section 3, we discuss and compare the specific case of connectivity and rigidity percolation on trees. In Section 4 we summarize the matching algorithms which may be used to find the percolating cluster in both connectivity and rigidity cases. The behavior in the connectivity and rigidity cases on site diluted triangular lattices is then compared. Section 5 contains a summary and discussion of the similarities and differences between connectivity and rigidity percolation in more general terms. 


\section{RIGIDITY AND CONNECTIVITY OF RANDOM GRAPHS}

In mathematical terms, percolation is the study of the connectivity properties of random graphs as a function of the number of edges in the graph. We are usually interested in the asymptotic limit of graphs with an infinite number of vertices. In the physics community, we usually study this process on a regular lattice (e.g. square, simple cubic, triangular) and consider the effect of adding or removing edges which lie between the vertices of these graphs. However the mean field limit is equivalent to considering connections between all sites on the lattice, no matter their Euclidean separation. The behavior of this "infinite range" model is equivalent to the limit of infinite dimensional lattice models (e.g. infinite dimensional hypercubes) and tree models. The reason for the equivalence of the critical behavior of these models is that they are all dominated by long range "rings", whereas on regular lattices in lower dimensions short range rings can be very important. Field theory models seek to add short range loops to these models in a systematic and non-perturbative (in some sense) way.

We consider lattices consisting of sites which are defined to have $z$ neighbors (i.e. they have coordination number $z$ ). We then adding edges between neighbors randomly with probability $p$. Once this process is finished we are left with a random graph with each site having average coordination $C=z p$. In studying percolation, we are always asking the question "Is it possible to transmit some quantity (i.e. a scalar, or a vector) across a graph". If it is possible to transmit the quantity of interest, we say that the network is above the "percolation threshold" $p_{c}$ (or equivalently $C_{c}=z p_{c}$ ) for that quantity. If it is impossible to transmit the quantity of interest we are below $p_{c}$. At $p_{c}$ the part of the random graph which transmits the quantity of interest is fractal provided the percolation transition is second order. The probability that a bond is on this "percolating backbone" is $P_{B}$, and is one of the key quantities in the analysis of percolation problems on regular lattices. If the percolation transition is "second order",

$$
P_{B} \sim\left(p-p_{c}\right)^{\beta^{\prime}} \text { as } p \rightarrow p_{c}^{+}
$$

with $\beta^{\prime}>0$. On trees it is more difficult to define $P_{B}$. Nevertheless we are able to analyze the problem effectively using "constraint-counting" ideas [7].

Let us for a moment put aside thinking in terms of percolation and instead develop constraint counting ideas originally discussed by Maxwell, and which have been developed extensively in the engineering, math and glass communities $[7,8]$. These ideas have not been applied to connectivity percolation till recently and are enriching to both the connectivity and rigidity cases. At a conceptual level constraint counting is deceptively simple. To illustrate this, we assign to each site of our lattices a certain number of degrees of freedom. In connectivity percolation, the transmission of a scalar quantity is of interest, therefore each site is assigned one degree of freedom. However if we consider a point object in two dimensions from the point of view of the transmission of forces, it has two degrees of freedom (two translations). In $d$ dimensions point masses have $d$ degrees of freedom. However extended objects have rotational degrees of freedom, so that when we have clusters which are mutually rigid, we must also consider these rotational degrees of freedom. We call such objects bodies and they have $d(d-1) / 2$ degrees of freedom. From a model viewpoint, we allow the number of degrees of freedom of a free site to be a control variable which we label $g$, with $g=1$ the connectivity case. With equal generality we may say that $G$ is the number of degrees of freedom of a rigid cluster or body. It is easy to see that there is a vast array of models with $g \neq 1$, and with $G \neq 1$ and much of the physics and applications of these models have yet to be examined [7].

Now we have a model in which a free site has $g$ degrees of freedom, so that a 
lattice with $N$ sites(and no edges) has a total of $F=N g$ degrees of freedom, or "floppy modes" (i.e. modes which have zero frequency due to the fact that there is no restoring force). Constraint counting consists in simply saying that each time an independent edge is added to the lattice, the number of degrees of freedom (zero frequency modes) is reduced by one if this edge is not "wasted" (see later). For example, the minimum number $E_{\min }$ of constraints needed to make a rigid cluster out of a set of $N$ sites is

$$
E_{\min }=N g-G,
$$

which holds for the case in which edges are put on the graph in such a way that none is wasted. In general, if $E$ edges are added to the graph, the number of degrees of freedom (or floppy modes) which remains is

$$
F=N g-E+R .
$$

Note the additional term $R$ on the right hand side. This term is key in understanding the relation between constraint counting and percolation, and in finding algorithms for rigidity percolation. $R$ is the number of "redundant bonds" or "wasted" edges which are added to the lattice. An edge does not reduce the number of floppy modes if it is placed between two sites which are already mutually rigid, in which case this edge is "redundant". The simplest examples of subgraphs containing a redundant bond on a triangular lattice are illustrated in Fig. 1 for the connectivity $(g=1)$ and $g=2$ rigidity cases.

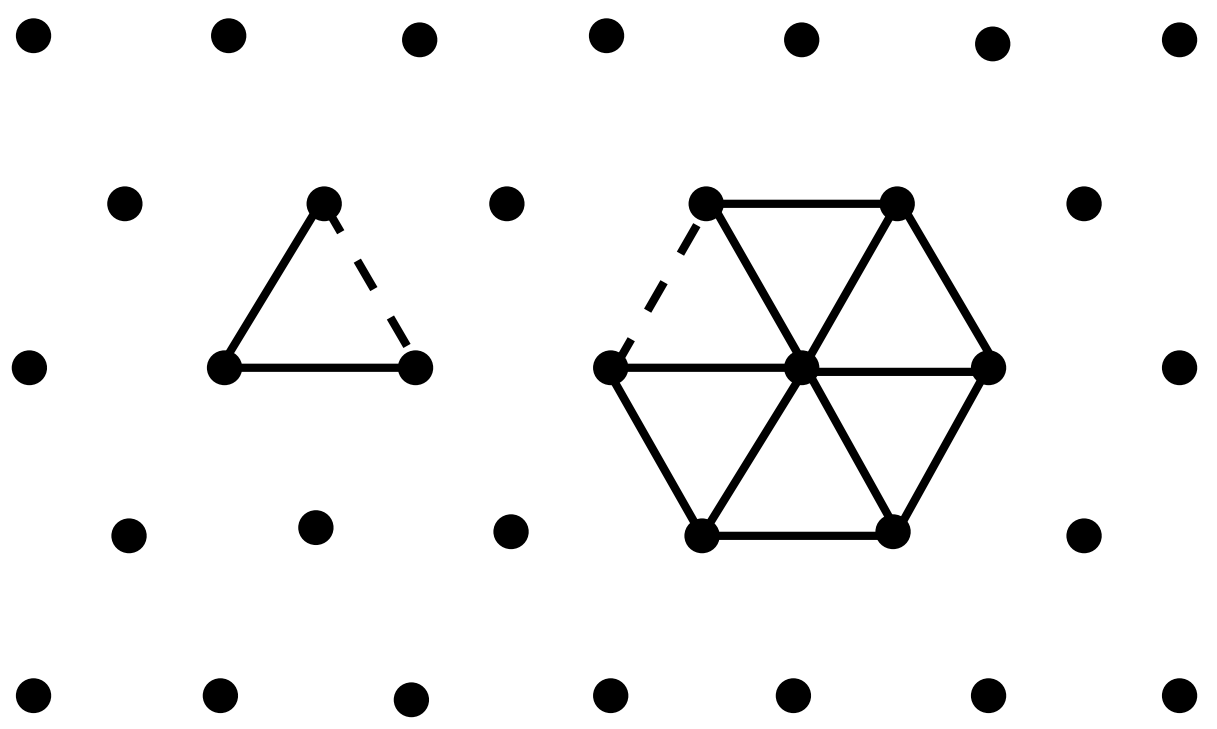

Figure 1: The simplest subgraphs on a triangular lattice which contain a redundant bond (dashed). Connectivity case (left), $g=2$ rigidity case (right).

Note that any one of the bonds in these structures could be labeled as the redundant one. However once any one of them is removed, all of the others are necessary to ensure the mutual rigidity of the structure. In fact the set of all bonds which are mutually redundant form an "overconstrained" or "stressed cluster". This is because, in the rigidity case we can think of each edge as being a central force spring, which means that there is a restoring force only in tension and compression. Then an overconstrained cluster of such springs (with random natural lengths) is internally stressed due to the redundant bond. In the connectivity case each bond is like a wire which can carry current or fluid flow. The simplest overconstrained cluster is then a loop which can support an internal "eddy" current. Rigid structures which contain no redundant 
bonds are minimally rigid or "isostatic". In connectivity percolation isostatic structures are trees, whereas in $(g>1)$ rigidity percolation isostatic structures must always contain loops (see Fig. 1).

In percolation problems, we are interested in the asymptotic limit of very large graphs $(N \rightarrow \infty)$, and it is more convenient to work with intensive quantities, so we define $f(p)=F / g N$ and $r(p)=2 R g / z N$ which leads to

$$
f(p)=1-\frac{z}{2 g}(p-r(p)),
$$

where the number of edges $E / N=z p / 2$. The normalization on $r(p)$ is chosen this way because $r(p)$ is now the probability that a bond is redundant (times $g$ ). Note that we normalize the number of floppy modes by $g$ to be consistent with previous work [5-7]. We have shown that $f(p)$ acts as a free energy for both connectivity and rigidity problems, so that if $\partial f(p) / \partial p$ undergoes a jump then the transition is first order. The behavior of this quantity is directly related to the probability that a bond is overconstrained $P_{o v}$ via the important relation [6]

$$
\frac{\partial f}{\partial p}=-\frac{z}{2 g}\left(1-P_{o v}\right)
$$

If the transition is second order, the second derivative $\partial^{2} f / \partial p^{2} \sim\left(p-p_{c}\right)^{-\alpha}$, where $\alpha$ is the specific heat exponent [6].

On both triangular lattices and on trees, we also calculate the infinite cluster probability. This is composed of the backbone plus the dangling ends. Dangling ends are rigidly connected to the backbone but do not participate in the transmission of the quantity of interest. Examples of dangling ends in the connectivity and rigidity cases are illustrated in Fig. 2.

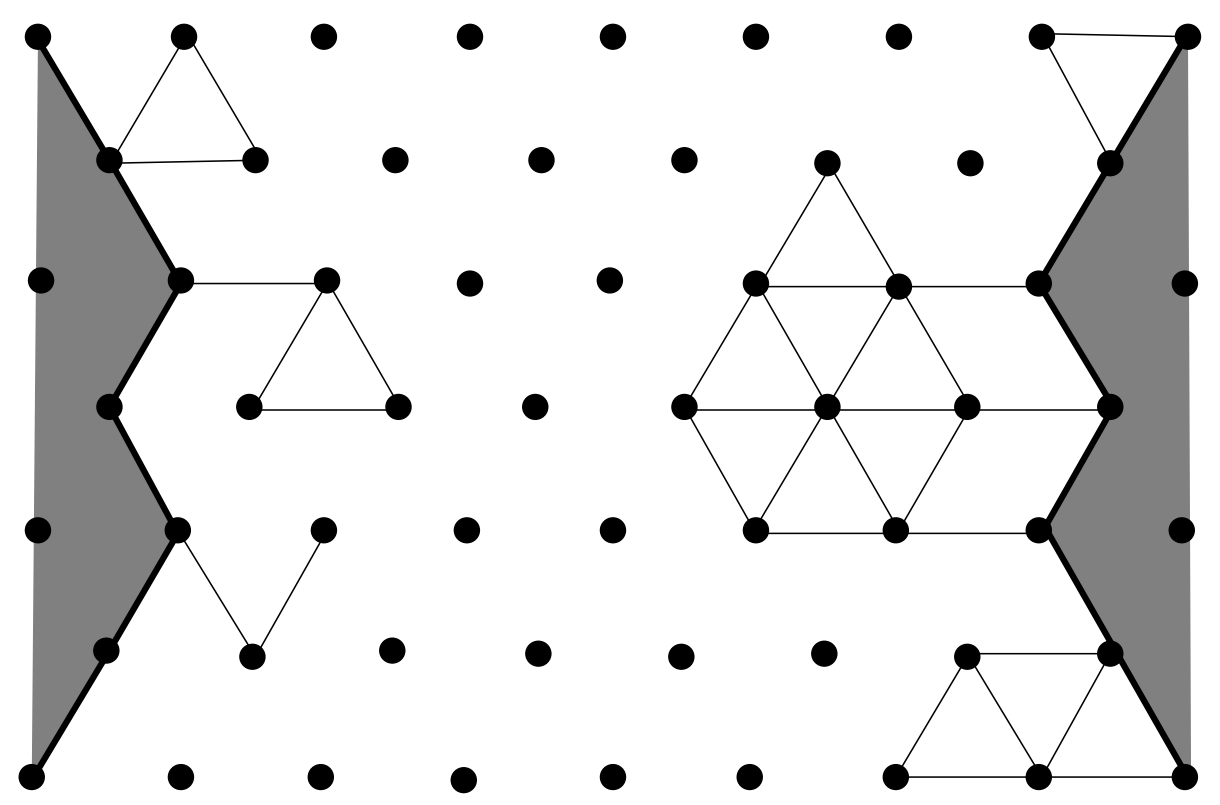

Figure 2: Examples of dangling ends (thin lines) connected to a backbone (shaded). Connectivity case (left) and $g=2$ rigidity case (right).

\section{TREES}

In our previous analysis of tree models, we have considered propagation of rigidity outward from a rigid boundary (this is the same as a strong surface field). Here we also add a bulk field which induces rigidity. The results are essentially the same whether the 
boundary or the bulk field is used. In percolation problems, the bulk field corresponds to nuclei embedded in the lattice and tethered to a rigid substrate. This is the sort of construction envisioned by Obukov [9], and used extensively in the construction of models for connectivity percolation. The mean-field equation for the order parameter on trees is then,

$$
T_{0}=h+(1-h) \sum_{l=g}^{z-1}\left(\begin{array}{c}
z-1 \\
l
\end{array}\right)\left(p T_{0}\right)^{l}\left(1-p T_{0}\right)^{z-1-l},
$$

where $T_{0}$ is the probability that a site is connected to the infinite rigid cluster through one branch of a tree (see the paper by Leath for more details on the derivation), and $h$ is the probability that a site is rigidly connected to the rigid substrate ( $h$ is sometimes called a "ghost field"). On trees, the probability that a bond is overconstrained is,

$$
\frac{N_{0}}{N_{B}}=T_{0}^{2}
$$

so that we have the key equation,

$$
\frac{\partial f}{\partial p}=-\frac{z}{2 g}\left(1-T_{0}^{2}\right) .
$$

Other useful formulae are the probability that a bond is overconstrained,

$$
P_{o v}=T_{0}^{2},
$$

and the probability that a bond is on the infinite cluster,

$$
P_{\text {inf }}=T_{0}^{2}+2 T_{0} T_{1} \text {. }
$$

In the connectivity cases, an infinitesimal field $h$ or any finite order parameter at the boundary is sufficient to allow a percolation transition to occur on trees. In contrast in $g \neq 1$ cases, there must be a finite $h$, or a finite boundary field before a transition occurs. These differences are illustrated in Fig. 3.
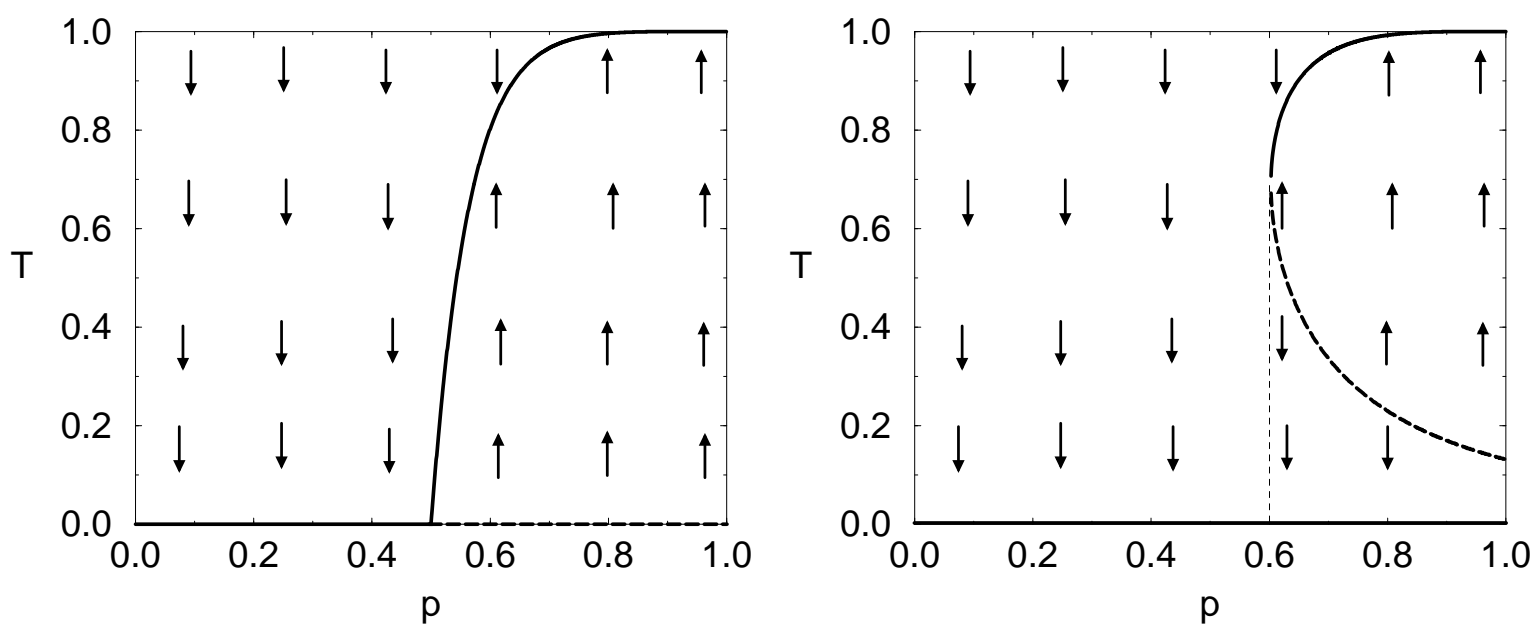

Figure 3: Domains of attraction of the mean field equations (6) with $h=0$ : (left) A typical connectivity behavior (this example is $z=3$ ); and (right) a typical rigidity behavior (this example is $z=6, g=2$ ). Dark lines are stable (attractive) solutions and shaded lines repulsive.

It is clearly seen from these figures that the rigidity transition is first order on trees, while the connectivity one is second order. The results presented in Fig. 3 are 
simple to obtain. We iterate the mean field equation (6) until a stable fixed point is reached (there are similar equations for $T_{1}$ etc.) and we then evaluate Eqs. (7) (9). We identify the point at which the stable solution becomes nonzero as $p_{s}$, the spinodal point, for reasons discussed below. In the connectivity case $p_{s}=p_{c}$ because the transition is second order.

We also want to find the total number of redundant bonds $r(p)$ and the total number of floppy modes $f(p)$. In order to find these quantities, we integrate Eq. (7) and then use Eq. (3). However, the integration of Eq. (7) leads to one free constant. This constant depends on the situation we wish to model. If we wish to model a regular lattice, then we impose the constraint,

$$
r(1)=1-\frac{2 g}{b z}
$$

for example in the case of central force springs on a triangular lattice $1 / 3$ of the springs are redundant when $p=1$. However on trees,

$$
r(1)=1-\frac{g}{b(z-1)}
$$

In using tree models to provide approximations to rigidity percolation on regular lattices, we impose the constraint (11) [7]. Then we find that $r(p)$ approaches zero at a critical point $p_{c}$, which is NOT the same a $p_{s}$ if $g>1$. However, if $g>1$, this $p_{c}$ is very close to the Maxwell estimate $p_{c}^{\text {Maxwell }}=2 g / b z$, and is the same as that found numerically for infinite range (random bond) models [7]. Typical results found for tree models are presented in Fig. 4.
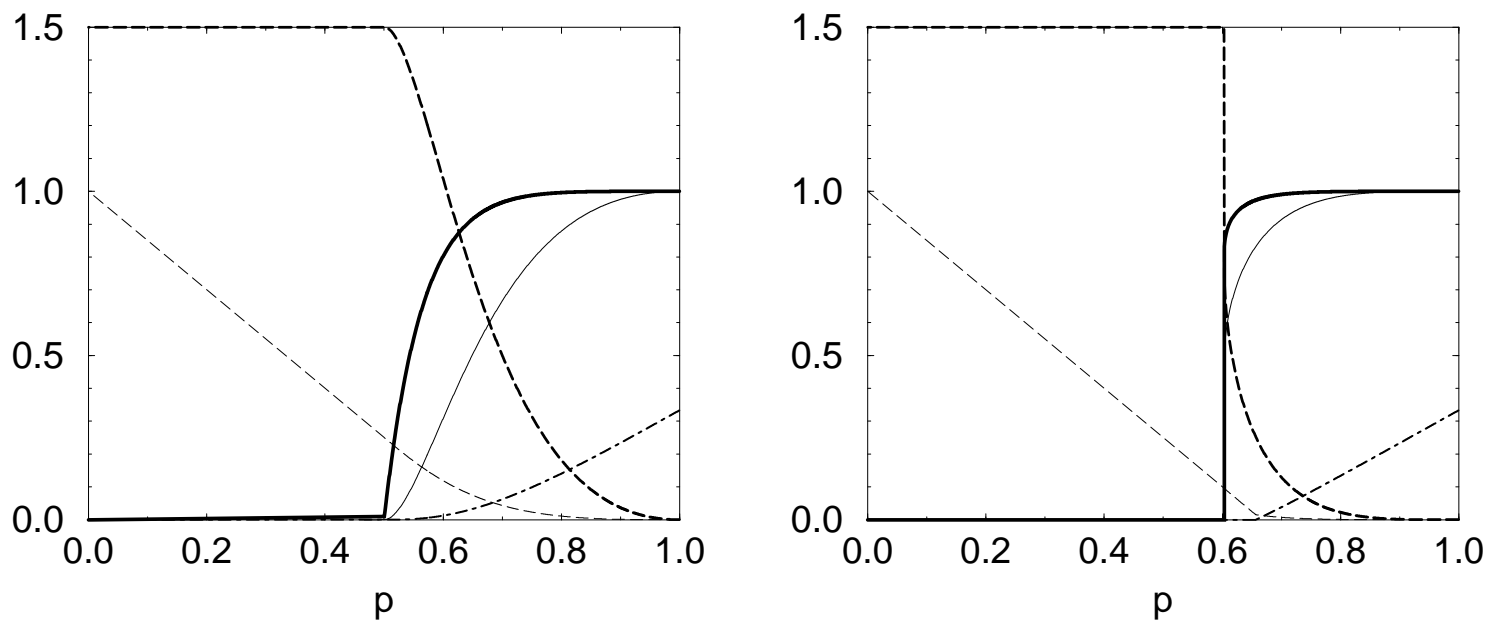

Figure 4: The order parameter, floppy modes and its derivative as a function of $p$ for a typical connectivity $z=3, g=1$ (left), and rigidity $z=6, g=2$ (right) cases, both with $b=1$. $f(p)$ (dashed), $r(p)$ (dot dashed), $-d f / d p$ (heavy dashed), $P_{o v}($ thin solid $), P_{\text {inf }}$ (heavy solid)

In the connectivity case we find $p_{c}=p_{s}$, while in rigidity cases $p_{s}<p_{c}$. For the rigidity case of Fig. $4, p_{s}=0.605$, while $p_{c}=0.655$.

\section{TRIANGULAR LATTICES}

We consider connectivity percolation $(g=1, b=1, z=6)$ and central force percolation $(g=2, b=1, z=6)$ on site diluted triangular lattices. In connectivity percolation the scaling behavior near the percolation threshold has been proven on trees and has been extensively tested on regular lattices using large scale numerical 
simulations. We have carried out a similar program for rigidity percolation as summarized below for triangular lattices. However doing numerical simulations in the rigidity case has required a breakthrough in algorithm development, and this has only occurred recently through contact with the mathematical computer science community. The methods developed for rigidity have even improved some aspects of algorithmic methods [12] for the connectivity case, as discussed below.
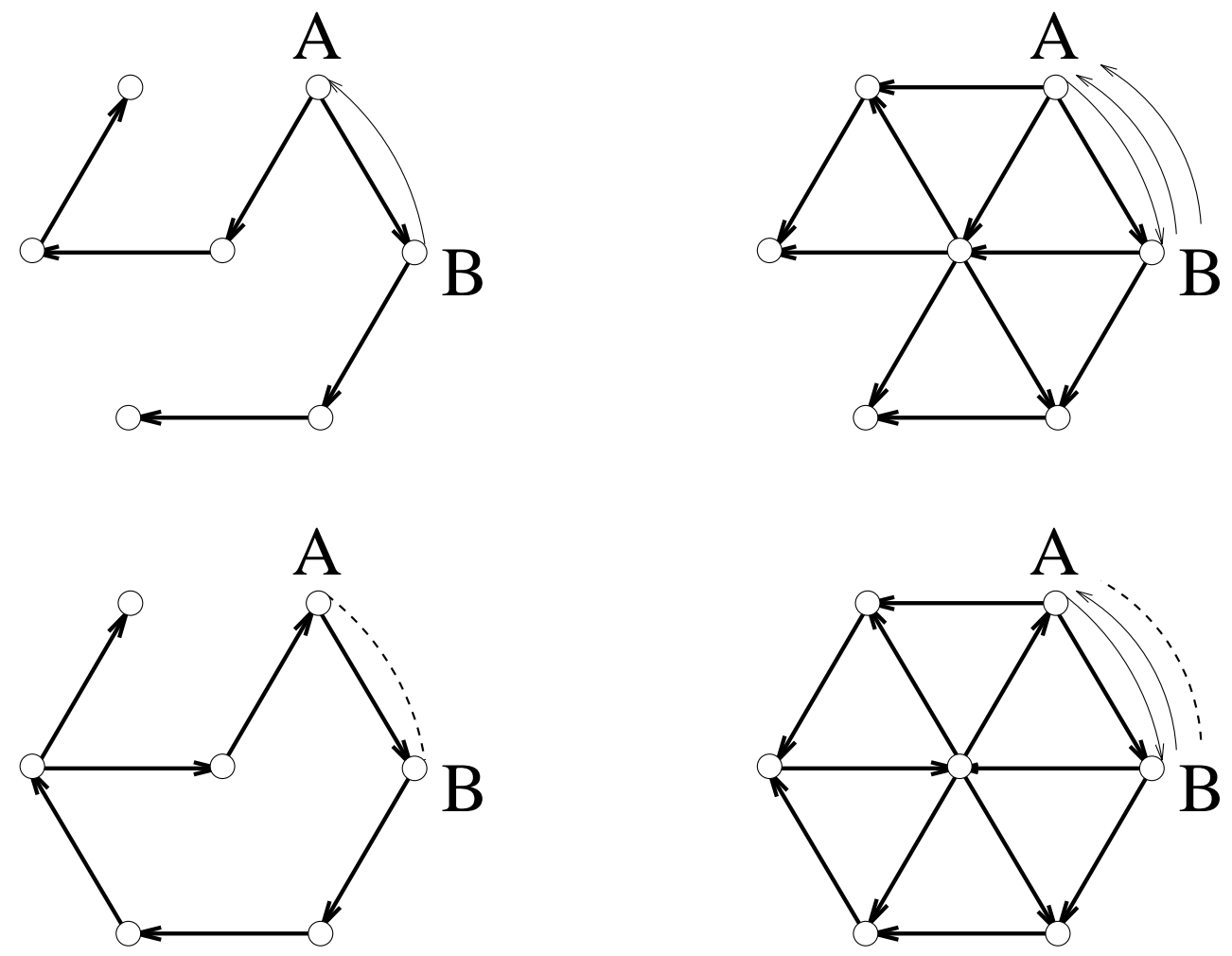

Figure 5: Examples of successful (top figures) and failed (bottom figures) matches in the connectivity ( $g=G=1$, left figures) and joint-bar rigidity ( $g=2, G=3$, right figures) cases on triangular lattices. AB is the new bond that is being tested. Each site has $g$ degrees of freedom and therefore accepts at most $g$ incoming arrows. Each new bond carries with it $G$ auxiliary arrows, which must also be matched. If any of the arrows cannot be matched (dashed), the new bond is redundant.

Before discussing the rigidity algorithms, it is important to point out the limitations of these methods. Firstly these algorithms are able to identify structures which can support stress (rigidity case), or which can carry a current (connectivity case). They do not find the actual current or stress, but rather those bonds which are able to transmit the load from an input node or set of nodes to an output node or set of nodes. In the connectivity percolation case it is trivial that the actual geometric realization of a given graph connectivity does not change the set of bonds which carry current from a fixed set of input nodes to a fixed set of output nodes. However in the rigidity case, there can be "special" realizations of a given graph which are responsible for singular rigid properties of "generically" rigid clusters. This may occur when there are "degenerate" constraints (e.g. parallel bonds on a lattice). The probability of such degenerate realizations is zero on geometrically disordered lattices and for this reason geometry, and the existence of these degenerate configurations, may be simply ignored in this case. In the mathematical community this problem is called "generic rigidity" and is the only one for which powerful algorithms exist. Thus the problem of rigidity on a regular triangular lattice remains unsolved. The results described below apply to triangular lattices whose sites have been randomly displaced (e.g. by 0.1 of a lattice 
constant). In addition, the powerful matching algorithms that we use apply to "jointbar graphs in the plane" and to a subset of graphs in general dimensions (so-called body - bar problems) However, it turns out that glasses in $3-d$ correspond to a case which is solvable (they map to a body-bar problem), so this is one of those unusual cases where the practical case is actually theoretically convenient.

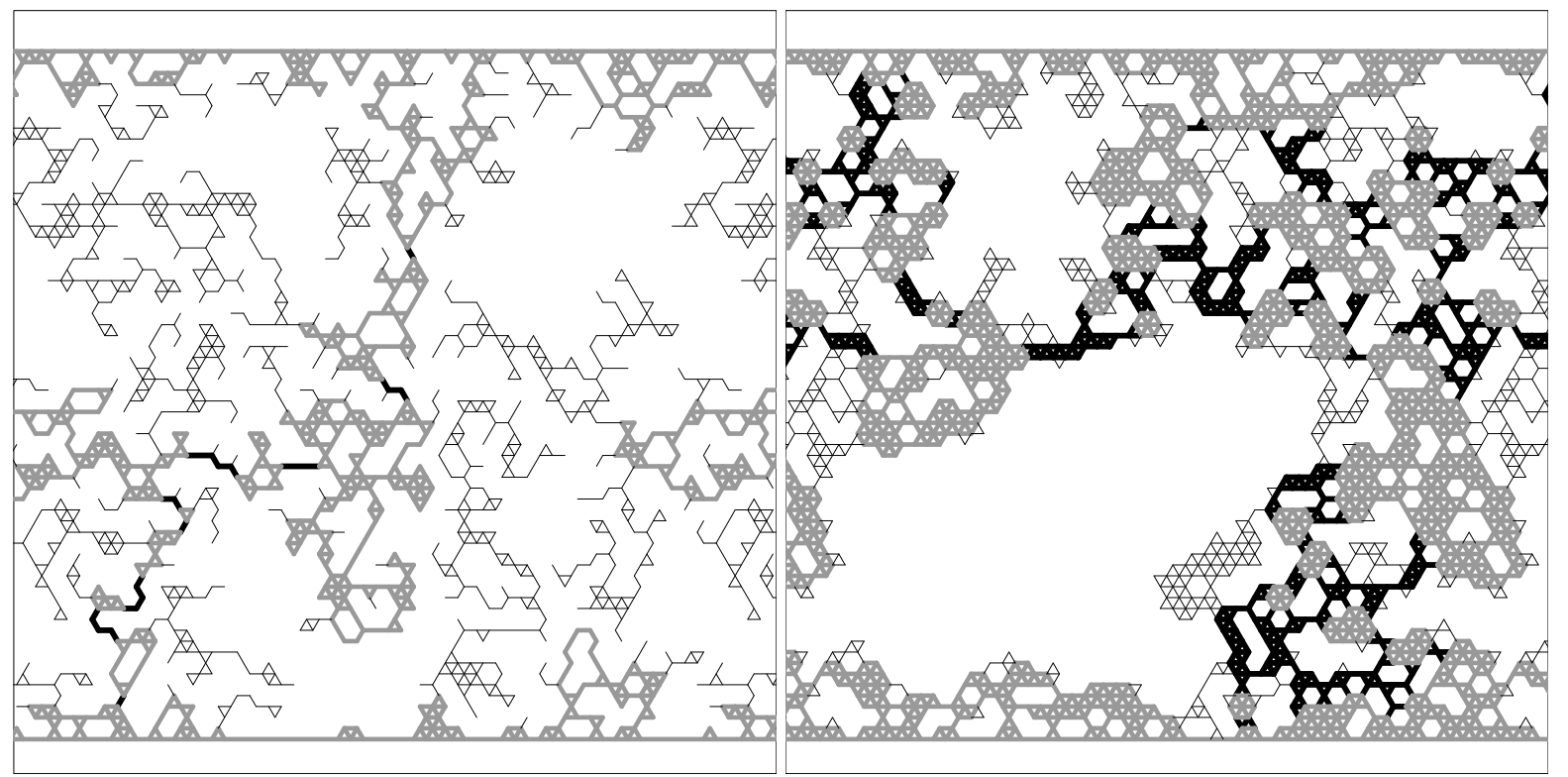

Figure 6: The infinite-cluster geometry for connectivity (left) and rigidity(right) percolation on site-diluted triangular lattices with $L=64$. In each case dark wide lines are cutting bonds, wide lines are non-critical backbone bonds (blobs) and thin lines are dangling ends.

The matching algorithm is implemented as follows [10]:

Start with an empty triangular lattice(no bonds) and assign to each node $g$ degrees of freedom.

Then:

1. Randomly add a bond to the lattice.

2. Test whether this bond is redundant with respect to the bonds which are currently in the lattice.

3. If the bond is redundant do not add it to the lattice, but instead store its location in a different array.

4. Return to 1.

End

The key step is step 2. The algorithm to do this test is rigorous and based on Laman's theorem. It was developed by Bruce Hendrickson, who also provided a key service in explaining his algorithm to the physics community. Step 2. is performed by exact constraint counting. This is implemented by "matching" constraints (bonds) to degrees of freedom, with the restriction that the constraints can only match degrees of freedom at each end of the corresponding bond. Thus it is natural to represent this constraint counting by using arrows to indicate the degree of freedom to which each bond is matched. The idea is that, when a new bond is added, it must be possible to match $G+1$ arrows to degrees of freedom of the graph in order for this new bond not to 
be redundant. If this task can be accomplished the new bond is independent, which means that it is not wasted, and is left on the graph. In this case only $G$ auxiliary arrows are removed. If on the other hand some of the new arrows cannot be matched, the last edge is redundant and all $G+1$ arrows are erased.

A successful and a failed match are illustrated in Fig. 5 for a connectivity $(\mathrm{g}=1)$ case and a rigidity $(\mathrm{g}=2)$ case. Note that the bond that is being tested carries with it $G$ additional "copies" which account for global degrees of freedom of a rigid cluster. In the connectivity case $G=1$, while on central-force bar-joint networks in two dimensions $G=3$.

A failed match occurs when a bond is unable to find a degree of freedom to "cover". This bond is then redundant. In trying to find a degree of freedom to which a redundant bond can be assigned or "matched", the algorithm identifies all bonds which are "overconstrained" of stressed with respect to that bond. This set of bonds is called a Laman subgraph. Note that if a redundant bond is already in a graph, it is not possible to add a new bond and test its redundancy with this method. This is the reason that the algorithm proceeds by adding bonds one at a time starting with an empty lattice. Any error is testing the redundancy of a bond invalidates the rest of the addition sequence. However since this algorithm is an integer method there is no problem with roundoff. It is easy to see that the matching algorithm is quite efficient, however if requires quite a bit of effort to fully optimize these methods.

Pictures of the infinite cluster for connectivity and rigidity percolation on a triangular lattice are presented in Fig. 6.
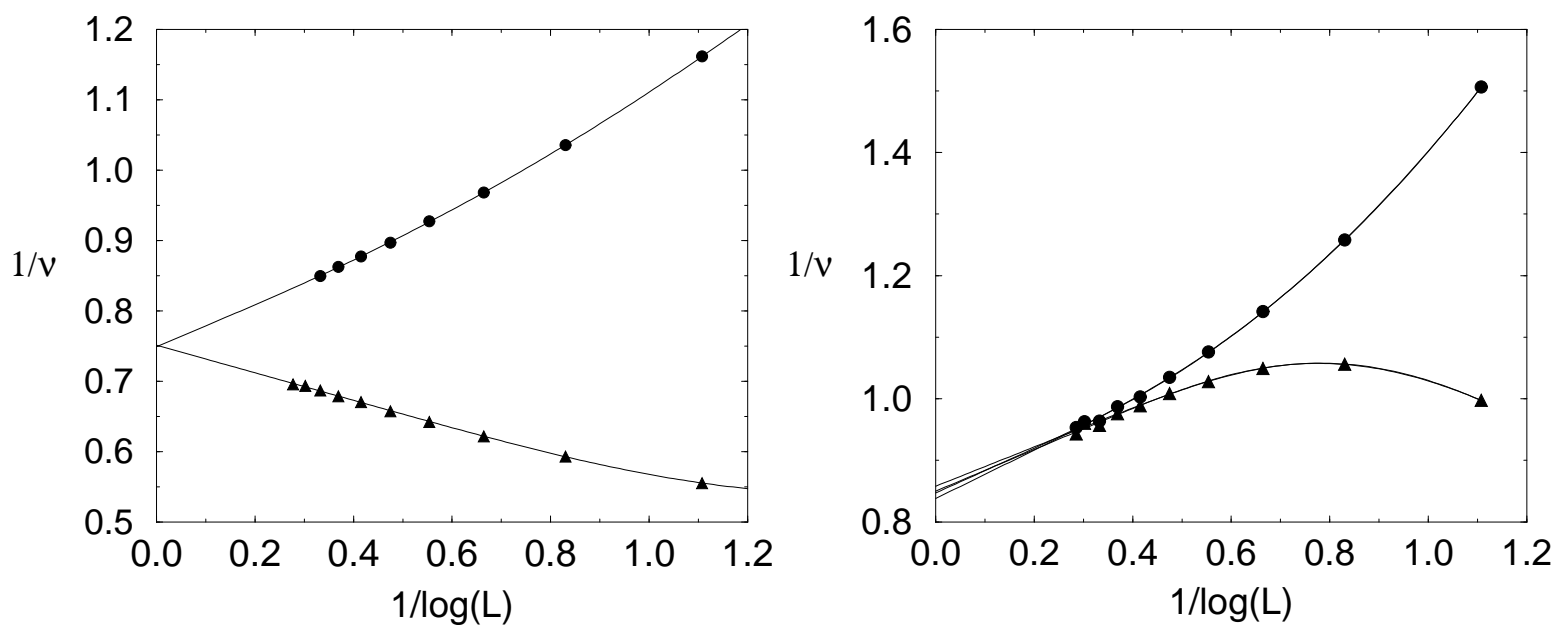

Figure 7: Analysis to find the correlation length exponent $\nu$ for connectivity (left figure) and rigidity percolation (right figure) on triangular lattices. In both figures the upper curve is a fit (including corrections to scaling) of $\delta p_{c} \sim L^{-1 / \nu}$ (full circles) while the lower curve is for the number of cutting bonds $n_{c} \sim L^{1 / \nu}$ (full triangles).

Because of the fact that we add bonds one at a time until the percolation point is reached, we are able to identify $p_{c}$ exactly for each sample, and therefore measure the components of the spanning cluster exactly at $p_{c}$. This eliminates the error associated with measurements at fixed values of $p$, since estimated exponents are known to depend very sensitively on $p$. This method was proposed and applied for the first time in ref. [5].

At $p_{c}$ we identify three different types of bonds: backbone bonds, dangling ends and cutting bonds. These together form the infinite cluster. The cutting bonds are stressed (belong to the backbone), but they are "critical" because if one of them is

\footnotetext{
${ }^{1} \mathrm{It}$ is valid to rearrange previously existing arrows, provided this is done in such a way that all remain matched to some degree of freedom
} 
removed, load is no longer transmitted across the infinite cluster. The results in Fig. 5 are for bus bars at the top and bottom of the figures and periodic boundary conditions in the other direction. In order to find the correlation length exponent, we used two relations: Firstly the size dependence of the threshold behaves as $\delta p_{c} \sim L^{-1 / \nu}$ and secondly, the number of cutting bonds varies as $n_{c} \sim L^{1 / \nu}[4]$. An analysis of this data in the connectivity and rigidity cases is presented in Fig. 7.

It is seen from this figure that the rigidity case has a different $\nu=1.16 \pm 0.03[5,6]$ than the connectivity case $\nu=4 / 3$. A finite-size-scaling plot of the infinite-cluster and backbone densities at $p_{c}$ is presented in Fig. 8, along with the density of dangling ends.
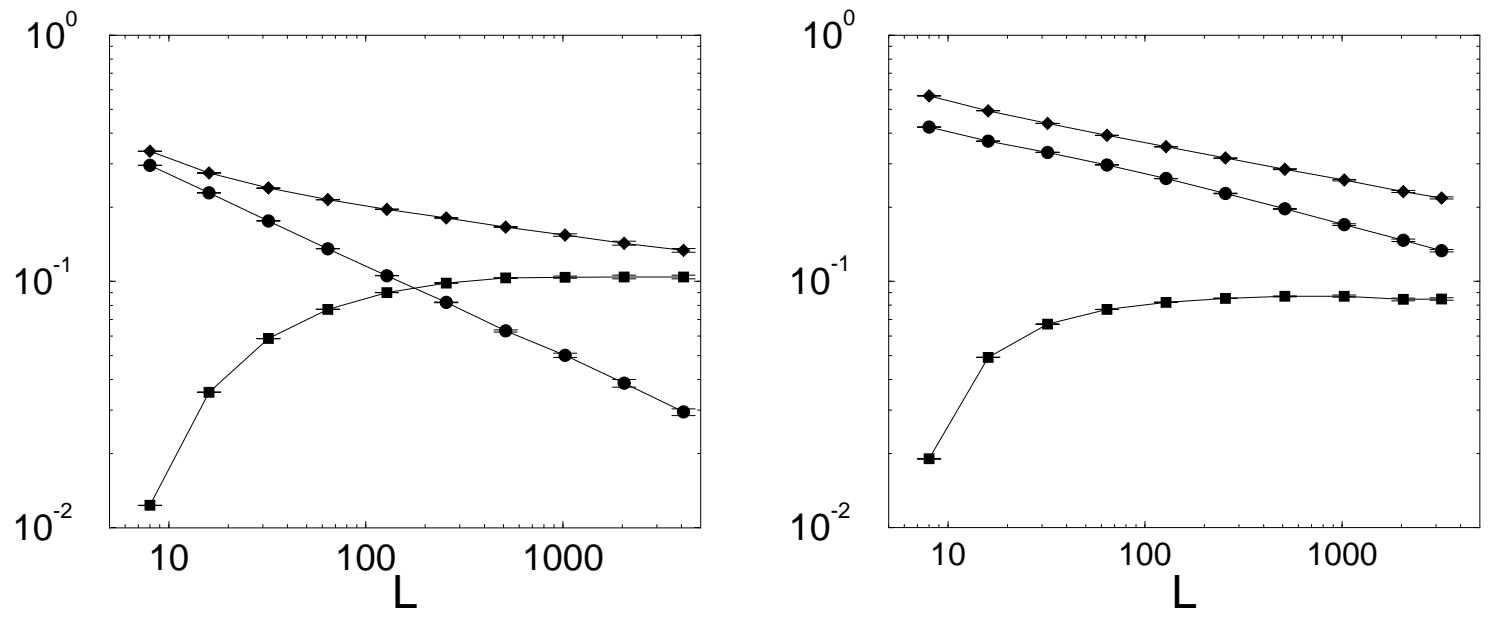

Figure 8: The density of backbone bonds(circles), infinite cluster bonds(diamonds) and dangling bonds(squares) for the connectivity ( $g=1$, left figure) and rigidity $(g=2$, right figure) cases on site diluted lattices. Our method of single-bond addition allowed these measurements to be made exactly at $p_{c}$ for each sample.

What is unambiguous from these figures is that the backbone density is decreasing algebraically $P_{B} \sim L^{-\beta^{\prime} / \nu}$, and from this data we find that in the rigidity case $\beta^{\prime}=0.25 \pm 0.02[5,6]$. It also appears that the infinite-cluster probability is decreasing algebraically, however that is difficult to reconcile with the behavior of the dangling ends. In the connectivity case much larger scale simulations have been done, and it is known that the algebraic decrease in the infinite cluster probability continues, so that the infinite cluster in the connectivity case is indeed fractal [4]. In the rigidity case, such large scale simulations are still lacking, so the possibility of an infinite cluster with finite density (about 0.1) remains.

\section{SUMMARY}

The development of tree models and the use of new algorithms in numerical studies have revolutionized our understanding of the geometry of rigidity percolation. In particular we now know that on triangular lattices, the rigidity transition is second order, but in a different universality class to the connectivity case. In contrast on trees the rigidity transition is first order while the connectivity transition is second order. The geometry of rigidity percolation is different from that of connectivity percolation due to the requirement of multiple connectivity in the rigidity case. However this is clearly not enough to ensure that the transition becomes first order. Perhaps a deeper question is whether the infinite cluster breaks up into two or an infinite number of subclusters when a critical bond is removed. In the connectivity case, the answer is clearly two. In the rigidity case it is infinity on trees and difficult to analyze precisely 
interesting class of problems.

\section{ACKNOWLEDGEMENTS}

PMD and CM thank the DOE for support under contract DE-FG02-90ER45418 and CM also acknowledges support from the Conselho Nacional de Pesquisa CNPq, Brazil.

\section{REFERENCES}

1. G. Deutscher, R. Zallen and J. Adler (Eds.), Percolation Structures and Processes, Bristol: Adam Hilger (1983)

2. G. Grimmett, Percolation, New York: Springer-Verlag (1989)

3. H. Kesten, Percolation Theory for Mathematicians, Boston: Birkhuser (1982)

4. D. Stauffer and A. Aharony, Introduction to Percolation Theory 2nd ed., London: Taylor \& Francis (1992)

5. C. Moukarzel and P.M. Duxbury, Phys. Rev. Lett. 75, 4055 (1995); C. Moukarzel, P.M. Duxbury and P.L. Leath, Phys. Rev. Lett. 78, 1480 (1997)

6. D. Jacobs and M.F. Thorpe, Phys. Rev. Lett. 75, 4051 (1995); D. Jacobs and M.F. Thorpe, Phys. Rev. E53, 3682 (1996)

7. C. Moukarzel, P.M. Duxbury and P.L. Leath, Phys. Rev. E55, 5800 (1997); P.M. Duxbury, D. Jacobs, M.F. Thorpe and C. Moukarzel, submitted to Phys. Rev. E.

8. J.C. Phillips, J. Non-Cryst. Solids 43, 37 (1981); M.F. Thorpe, J. Non-Cryst. Sol. 57, 355 (1983)

9. S. Obukov, Phys. Rev. Lett. 74, 4472 (1994)

10. B. Hendrickson, Siam J. Comput. 21,65 (1992); C. Moukarzel, J. Phys. A29, 8079 (1996)

11. Comment to PRL by D. Jacobs and M.F. Thorpe, and reply by P.M. Duxbury, C. Moukarzel and P.L. Leath. to appear in the June 15th issue of Phys. Rev. Lett.

12. C. Moukarzel 1997, Int. J. Mod. Phys. C, to appear. 